\title{
Felix Platters Stellung in der Medizin seiner Zeit*
}

\author{
Von Huldrych M. Koelbing
}

1

Im Alter von nahezu 78 Jahren ist der Basler Medizinprofessor und Stadtarzt Felix Platter 1614 verstorben ${ }^{1}$ - hundert Jahre nach der Geburt seines großen Vorbildes Andreas Vesalius. Während dieser hundert Jahre hat die Medizin tiefgreifende Umwandlungen erfahren; Felix Platter hat sie mit lebhaftem Geist wahrgenommen und selber tätig daran mitgewirkt. Aber wie groß ist sein Anteil an diesen Veränderungen? Welche Rolle hat er in jener Renaissance der Heilkunde gespielt? Die Frage ist leichter getan als beantwortet, und es besteht in der Würdigung Platters ein auffallender Unterschied zwischen den Autoren, die allgemeine Medizingeschichte schreiben, und denjenigen, die sich speziell mit Platter und der Stadt seines Wirkens befassen. Für die ersteren, z.B. Garrison ${ }^{2}$ oder Diepgen ${ }^{3}$, ist Felix Platter eine ausgesprochene Nebenfigur, die man weglassen könnte, ohne daß sich am Gesamtbild etwas ändern würde. In den Augen der letzteren jedoch erscheint er als Arzt und Gelehrter von weit mehr als nur lokaler Bedeutung; ich denke dabei vor allem an Albrecht Burckhardts Basler Fakultätsgeschichte ${ }^{4}$ und an Jean Karchers Platter-Biographie ${ }^{5}$. Der Unterschied in der Wertung ist zu groß, um in der bloßen Verschiedenheit der Maßstäbe - dort medizinische Weltgeschichte, hier Lokalhistorie seine hinreichende Erklärung zu finden. Entweder wird Platter von den Basler Medizinhistorikern überschätzt, oder er ist von den Autoritäten der allgemeinen Medizingeschichte bisher unterschätzt worden!

Internationale Anerkennung durch die Nachwelt hat Felix Platter vor allem als Psychiater gefunden (ich verweise auf AcKerknechts Kurze Geschichte der Psychiatrie ${ }^{6}$ ), sodann als Anatom und im Zusammenhang da-

* Vortrag, gehalten in der Schweizerischen Gesellschaft für Geschichte der Medizin und Naturwissenschaften am 10. Oktober 1964 in Zürich.

1 Vgl. H.Buess, Ein Basler Gedenktag als Auftakt zum Internationalen Kongreß für Geschichte der Medizin, National-Zeitung (Basel) Nr. 405, 3. September 1964.

${ }^{2}$ F.H.Garrison, An Introduction to the History of Medicine, 4. Auflage (1929), Neudruck, Philadelphia 1960.

3 P.Diepgen, Geschichte der Medizin, 1. Band, Berlin 1949.

4 A.Burckhardt, Geschichte der Medizinischen Fakultät zu Basel 1460-1900, Basel 1917.

5 J. Karcher, Felix Platter, Lebensbild des Basler Stadtarztes, 1536-1614, Basel 1949.

${ }^{6}$ E.H.Ackerknecht, Kurze Geschichte der Psychiatrie, Stuttgart 1957, S. 27. 
mit als Physiologe des Auges und schließlich, unabhängig von seinen medizinischen Leistungen, als Memoirenschreiber. Seine Jugenderinnerungen, in denen die Studienjahre (1552-1557) in Montpellier den breitesten Raum einnehmen, geben ein höchst lebendiges Bild jener Zeit, wie ein aufgeweckter Student aus dem Bürgerstand sie erlebte. Sie sind zuletzt 1961 in der englischen Ausgabe von JenNetr $^{7}$ wieder veröffentlicht worden; immer noch fehlt jedoch eine einwandfreie deutsche Edition, wie wir sie für die Lebensbeschreibung von Felix' Vater, Thomas Platter, in der vorbildlichen Ausgabe Hartmanns ${ }^{8}$ seit zwanzig Jahren besitzen.

Gelesen zu werden verdient der Essay des Zürcher Literaturhistorikers Fritz Ernst über Die beiden Platter (1927) ${ }^{9}$. Ernst faßt die Lebensgeschichte des Vaters und des Sohnes als ein zusammengehörendes Ganzes auf: Alles Wünschen, Streben und Sorgen des Vaters Thomas, des vom Schicksal in jungen Jahren so hart angefaßten Walliser Bergbauernbuben, habe in Felix, dem Sohn - nomen est omen - seine glänzende Erfüllung gefunden. Ein Satz, den Ernst über Felix Platters Stellung in der Medizin seiner Zeit schreibt, gibt knapp und treffend das bisher geltende Urteil der allgemeinen Medizingeschichte wieder ${ }^{10}$ : «Ein Bahnbrecher war er nicht, aber ging doch vorn im Zuge mit den andern.»

\section{2}

Ernsts Dictum ist einer jener Sätze, die man gerne gerade so, wie sie sind, von der früheren Generation übernehmen und weitergeben möchte, weil darin das Wesentliche so klar, einfach und einleuchtend ausgesprochen zu sein scheint. Aber besser ist es, solche Sätze von Zeit zu Zeit neu auf ihre Richtigkeit zu prüfen. Um dies zu tun, müssen wir uns nun einen Überblick über Platters Leistungen verschaffen, dann die Grenzen seines Denkens und Tuns diskutieren und schließlich seinen Einflu $\beta$ auf die Ärzte des späten 16. und des 17.Jahrhunderts abzuschätzen versuchen.

7 S.Jennet, Beloved Son Felix. The Journal of Felix Platter ..., translated and introduced by S. J., London 1961. - Das Buch enthält den Bericht über Platters Reise nach Montpellier, seine dortigen Studienjahre und die Heimreise, ist also keine vollständige Ausgabe der Lebensbeschreibung.

8 Th. Platter, Lebensbeschreibung, herausgegeben von A.Hartmann, Basel 1944.

${ }^{9}$ F.Ernst, Die beiden Platter, Neue Schweizer Rundschau 20 (1927) 1097-1111.

10 l.c., S. 1104. 
1. Als erstes Werk gab Felix Platter mit 47 Jahren (1583) seine Anatomie mit ihren fünfzig Bildtafeln heraus, das Buch De corporis humani structura et $u s u^{11}$. Es ging ihm dabei vor allem darum, die Vesalische Anatomie zu popularisieren, die «kaum zu übertreffenden» bildlichen Darstellungen der Fabrica VeSALS ${ }^{12}$ in etwas reduziertem Umfang und in handlicherer und billigerer Form zugänglich zu machen. Platter hat seine Anschauung aber nicht nur aus Vesal, sondern auch aus über fünfzig (meist freilich wohl nicht vollständigen) eigenen Sektionen gewonnen. Er berücksichtigt in seinem Lehrbuch auch die seit vierzig Jahren von der anatomischen Forschung gemachten Fortschritte. So ist ihm der Lungenkreislauf aufgrund der Beschreibung CoLombos (1559) bekannt; als Aufgabe der Lunge bezeichnet er es, das Blut von «Exkrementen» zu reinigen und ihm Luft beizumischen ${ }^{13}$. Im Bereich des usus partium, der physiologischen Funktion morphologischer Strukturen, enthält das Buch mindestens die eine, wichtige, neue Erkenntnis, daß im Auge die Bilder der Außenwelt von der Netzhaut (nervus retiformis) aufgenommen werden und nicht von der Linse (humor crystallinus), wie man das seit den Zeiten der alexandrinischen Anatomen immer angenommen hatte ${ }^{14}$.

2. Rund zwei Jahrzehnte später erschien Felix Platters Hauptwerk, die drei Bände der Praxis medica (1602-1608) ${ }^{15}$. Platter zeigt sich darin als ein Arzt, der die antike Tradition gut kennt und sie auch anerkennt, soweit sie ihm in Vernunft und Erfahrung begründet erscheint, der sie jedoch ablehnt, wo ihn kritisches Nachdenken und eigene Beobachtungen einen anderen Weg führen ${ }^{16}$.

11 F.Platter (Platerus), De corporis humani structura et usu libri III, Basel 1583 (Froben). Das 3. Buch (Bildtafeln) besitzt ein eigenes Titelblatt mit dem Druckjahr 1581. Unveränderter Neudruck (mit nur 48 statt 50 Bildtafeln) 1603 (König).

12 «...cum nemo hactenus rectius Vesalio figuras anatomicas depingi curaverit et vix dum etiam meliores illis effingi possint ...»-op. cit., Vorrede zum 3. Buch.

13 op. cit., S. 172.

14 op. cit., S. 187.

15 F.Platter, Praxeos seu de cognoscendis, praedicendis, praecavendis curandisque affectibus homini incommodantibus (sc. tractatus III), Basel 1602/03/08 (Waldkirch). Die Bände I und III existieren auch mit Druckjahr 1609; für den 1.Band handelt es sich nach A.Burckhardt (op. cit. sub no. 4) um einen Neudruck. Weitere Auflagen des Gesamtwerkes: 1625, 1656, 1666, 1736 bei anderen Basler Verlegern.

16 Vgl. das Widmungsschreiben der «Praxis» an den Herzog Friedrich I. von Württemberg: «... unice mihi fuit propositum veritatem quantum possem sectari nulliusque in verba magistri iurare; quae rationibus firmis experientiaque certā vera esse comperi, pro veris asseverare ...» (Hervorhebung durch K.). 
Um die damals schon gewaltige Fülle des Stoffes zu gliedern, hat Platter - Sie wissen es - ein neues Einteilungsprinzip ersonnen: Er ordnet die Krankheiten nicht mehr in erster Linie nach topographischen, sondern nach symptomatologischen Gesichtspunkten: Funktionsstörungen, Schmerzen (mit Einschluß der Fieber, die ja auch varias molestias verursachen) und morphologische Veränderungen (vitia) sind die Hauptthemen der drei Bände. Diese Ordnung erweist sich als sehr günstig für die Besprechung krankhafter Vorgänge, die den ganzen Organismus betreffen oder doch nicht nur auf ein einzelnes Organ beschränkt sind. So erfahren die Krankheiten und Störungen der Geistestätigkeit (laesiones mentis) eine imposante Gesamtdarstellung; gleicherweise können die Sehstörungen im Zusammenhang besprochen werden, unbekümmert darum, ob sie im Hirn, im Sehnerven oder im Auge selbst ihre Ursache haben. Andererseits werden so verschiedene Dinge wie Lähmungen und Knochenbrüche im gleichen Kapitel behandelt, da sie sich beide in einer Störung der willkürlichen Bewegung äußern; die Krankheiten der einzelnen Organe, etwa der Augen oder der Ohren, müssen wir dagegen in allen drei Bänden zusammensuchen, was für uns organspezialistisch verbildete Mediziner des 20. Jahrhunderts ungewohnt und mühsam ist. Näher als Platters Systematik steht unseren Denkgewohnheiten die ältere von Jean Fernel, in welcher die allgemeine Pathologie (Krankheitsursachen, Symptome, Puls, Fieber) der a capite ad calcem angeordneten speziellen Krankheitslehre vorangeht.

3. Die Praxis medica findet ihre Ergänzung in den nun wirklich aus dem vollen Leben gegriffenen Observationes; diese kamen im Todesjahr Platters heraus, also 1614, und ihr erster Teil liegt jetzt seit einem knappen Jahr, in der von BuEss bearbeiteten Übersetzung GoLDschmidts, auch auf deutsch vor ${ }^{17}$. Selbstverständlich - so muß man fast sagen - finden sich unter diesen Krankengeschichten einzelne Erstbeschreibungen, so der von MANI entdeckte Bericht über einen morbus haemolyticus neonati ${ }^{18}$. (Noch Karcher konnte - vor fünfzehn Jahren - die Plattersche Erstbeschreibung des «Thymustodes» rühmend erwähnen, wenn auch mit der Einschränkung:

17 F.Platter, Observationum in hominis affectibus plerisque corpori et animo ... incommodantibus libri tres, ad Praxeos tractatus tres accommodati, Basel 1614. (Waldkirch). - Observationes, Krankheitsbeobachtungen in drei Büchern. I.Buch: Funktionelle Störungen des Sinnes und der Bewegung. Übersetzt von G. Goldschmid, bearbeitet und herausgegeben von H.Buess, Bern 1963 (= Hubers Klassiker der Medizin und der Naturwissenschaften, Band 1).

18 N.MANI und L.Holländer, Frühe Schilderungen des Morbus haemolyticus neonatorum in Basel, Schweiz.med.Wschr. 89 (1959) 439-441. 
«Die richtige Deutung des Falles blieb ihm versagt. ${ }^{19}$ Seitdem sich aber der «Thymustod» als gelehrtes Hirngespinst erwiesen hat, stehen wir wieder etwas verlegen vor dieser Beschreibung eines kindlichen Todesfalles aus unklarer Ursache. - So können auch vortreffliche «Erstbeschreibungen» durch den Fortschritt der Wissenschaft ihren Rang und Glanz unversehens wieder verlieren.

Eindrücklicher als alle Einzelheiten ist es für uns, aus den Observationes (und auch aus der Praxis) zu erkennen, wie konsequent Platter bemüht war, durch die Autopsie Aufschluß über Sitz und Ursache der Krankheiten zu gewinnen. Die Anatomie war für Platter tatsächlich zum Fundament der Medizin geworden, zu einem Fundament, auf dem die Pathologie nicht weniger als die Physiologie aufgebaut werden mußte. In dieser Sache ist Felix Platter wirklich ein Pionier gewesen (vgl. Buess ${ }^{1}$ ) und damit - mit BenIvieni, Schenck von Grafenberg und Bonet - zu einem Wegbereiter Morgagnis geworden.

4. Es bleibt nun - von den postum erschienenen, unbedeutenden Quaestiones medicae ${ }^{20}$ abgesehen - noch Platters Peststatistik ${ }^{21}$ zu erwähnen. Darin hat der 75 Jahre alte Stadtarzt für die Basler Pest- und Fleckfieberepidemie von 1609 bis 1611 Haus für Haus alle Erkrankten und Verstorbenen aufgezeichnet. Dieses handschriftliche Dokument hat erst seit 1880 die ihm gebührende Beachtung gefunden; früher ist es wohl eher als Kuriosum denn als wissenschaftlich wertvolle Schrift betrachtet worden.

1. Wir kommen nun zu den Grenzen in Felix Platters Tun und Denken. Sie werden gerade in seinem Verhalten der Pest gegenüber besonders deutlich. Er, der medizinische Chronist von sieben Epidemien in Basel, der auch in den schlimmsten Zeiten seine Patienten gewissenhaft betreut hat,

19 J. Karcher, op. cit. sub no. 5, S. 83. Platter reiht den Fall unter die Atemstörungen ein: Beob. 161, S. 133 in der Ausgabe Goldschmidt-Buess.

20 F.Platter, Quaestionum medicarum paradoxarum et endoxarum centuria posthuma, als Anhang zur «Praxis» erstmals von Th. Platter II 1625 herausgegeben.

${ }^{21}$ F. Platter, Siben regierende Pestelentzen oder Sterbendt ze Basel ..., MS Universitätsbibliothek Basel. Verfaßt 1611, vgl. J. KARCHER, op. cit. sub no. 5, S. 77. Auszugsweise gedruckt: a) «Aus F.P.s Bericht über die Pest zu Basel ...», Festgruß der Universitätsbibliothek ..., Basel 1880; b) Rose Hunziker, Felix Platter als Arzt und Stadtarzt in Basel, Diss. Basel 1938, publ. Zürich 1939. 
versagte als Amtsarzt insofern, als er sich nicht energisch dafür einsetzte, durch eine strikte Quarantäne die Stadt vor der Pest zu schützen. Dieses Versagen wiegt um so schwerer, als der Erfolg der Maßnahme von den italienischen Städten schon längst praktisch erprobt worden war; zudem wußte Platter, daß die Krankheit von Reisenden aus verseuchten Gebieten eingeschleppt werden konnte ${ }^{22}$. Aber nicht weniger war er auch davon überzeugt, daß die Pest eine göttliche Heimsuchung sei, der man sich demütig zu beugen hatte. Im übrigen muß zu seiner Entlastung festgehalten werden, daß Platter als Archiater nur Berater der Obrigkeit war, und KARGHER hat darauf hingewiesen ${ }^{23}$, daß sich seine Amtstätigkeit zur Hauptsache im Schoße zahlreicher Kommissionen abwickelte. Absperrmaßnahmen, die den Handel beeinträchtigt hätten, waren aber den Basler Kaufleuten und Gewerbetreibenden ein Greuel; die mit Sicherheit zu erwartenden finanziellen Verluste scheinen ihnen mehr Schrecken eingejagt zu haben als der bloß wahrscheinliche Verlust des Lebens durch die herannahende Pest, und Felix Platter hat sich offenbar derartigen Erwägungen ohne ernsthaften Widerstand gefügt.

2. Auch die Grenzen von Platters Psychiatrie sind offensichtlich. Er betrachtete die Geisteskrankheiten zur Hauptsache als natürliche Affektionen und übertraf alle früheren Autoren an klinischer Beobachtungstreue (АкKERKNECHT ${ }^{6}$ ); aber er räumte doch auch dem Teufel und den bösen Geistern noch ihren Platz in der Ätiologie der geistigen Störungen ein. Das erscheint uns als bedauerliche Voreingenommenheit; hätte Platter jedoch die Möglichkeit der Besessenheit verneint, so wäre er seinen Zeitgenossen als unrealistisch, ja als selber verrückt erschienen. In der Behandlung der Irren verlegte Platter das Schwergewicht aus dem magischen in den medizinischen Bereich; aber er brach nicht ganz mit der mittelalterlichen Tradition: Er befreite einzelne Kranke aus ihren Gefängniszellen, aber er befreite nicht alle ${ }^{24}$. Platter war noch kein PineL, und der Geist der Renaissance war noch nicht der Geist der Aufklärung. Ähnliches wie für die Psychiatrie ließe sich auch für andere Seiten von Platters ärztlichem Denken und Wirken zeigen: Altes und Neues verbanden sich darin.

22 «Praxis» II, Spalte 75 der 3. Auflage (1656 - Titel des Werkes in dieser Auflage: FeLIcis Plateri ... Praxeos medicae tomi tres): «... aliqui ab infectis locis in alia loca, ubi nulla est pestis, fugientes ... post multos saepe dies peste primum corripiantur vel non correpti alios inficiant. »

23 J.KARcher, op. cit. sub no. 5, S. 54.f., 75.

24 J.KARCHER, op. cit., S. 70. 
3. Platters Grenzen in der Forschung lassen sich besonders hübsch an seinen Ansichten über die Augenlinse und den grauen Star demonstrieren ${ }^{25}$. Bis ins 18. Jahrhundert hinein wurde die Katarakt nicht als eine Trübung der Linse, sondern als eine Trübung im humor aqueus, zwischen Linse und Pupille, aufgefaßt. Den Sitz des Stars in der Linse selbst zu erkennen, war so lange unmöglich, als man diese für das eigentliche Sehorgan, den bildempfangenden Teil des Auges hielt. Denn wenn sie das war, so war sie unentbehrlich, und ein Auge konnte nur so lange überhaupt etwas sehen, als die Linse darin intakt war. Ein vom grauen Star befallenes Auge konnte jedoch, wie die Erfahrung lehrte, wieder zum Sehen gebracht werden, wenn man mit der Starnadel die Trübung aus dem Bereich der Pupille entfernte. Also konnte die Katarakt nicht eine Affektion der Linse sein.

Indem Felix Platter nun der Realität entsprechend die Netzhaut zum bildaufnehmenden Teil des Auges erklärte ${ }^{14}$, wertete er in Tat und Wahrheit die Linse zu einem entbehrlichen optischen Hilfsmittel ab. Zudem hatte er bei der Sektion von Augen alter Leute Trübung und Verhärtung der Linse als Ursache schlechten Sehens erkannt ${ }^{26}$, und schließlich sagt er von der Katarakt (er benützt den lateinischen Namen suffusio), sie sei ein kleines, linsengroßes Gebilde - «exigua tantum et vix lentis instar ampla materia ${ }^{27}$. Diese Aussage bezieht sich wahrscheinlich auf eine Beobachtung in vivo: Es kam gelegentlich vor, daß bei der Staroperation die trübe Masse nicht wie beabsichtigt hinter der Iris nach unten verschwand, sondern daß sie durch die Pupille in die Vorderkammer trat, wo sie natürlich dann gut sichtbar war. Solche Befunde haben zu Beginn des 18. Jahrhunderts bei der Aufklärung der Starpathologie tatsächlich eine Rolle gespielt. Felix Platter aber war, in den ersten Jahren des 17. Jahrhunderts, noch nicht imstande, sie richtig zu deuten und das zu sehen, was er - von unserem Standpunkt aus beurteilt - eigentlich hätte sehen müssen: nämlich, daß der graue Star nichts anderes ist als die trüb gewordene Linse. Seine Beobachtungen - es waren gute, richtige Beobachtungen - vermochten nicht, den Bann der Tradition zu brechen, für welche die Unversehrtheit der Augenlinse eine unabdingbare Voraussetzung für das Sehen war. Mit Platters «Abwertung des humor crystallinus» (wie man es nennen könnte) hatte diese

25 Näheres darüber ist in meiner Studie «Zur Renaissance der Ophthalmologie (um 1550 bis um 1630) mit besonderer Berücksichtigung der Schweiz» zu finden, deren Publikation zurzeit vorbereitet wird.

${ }^{26}$ F. Platter, Praxis I, 3.Auflage (1656), Spalte $192 \mathrm{f}$.

${ }^{27}$ l.c., Spalte 200. 
These zwar ihre logische Berechtigung eingebüßt - trotzdem hielt Platter daran fest. Deshalb kam er in der Pathologie des grauen Stares zu keinen neuen Erkenntnissen. Freilich: Wenn er tatsächlich den wahren Sachverhalt entdeckt hätte, so hätte ihm aller Wahrscheinlichkeit nach niemand geglaubt. KaSPar BAuHin, Platters jüngerer Fakultätskollege, lehrte auch die Physiologie des Auges wieder getreu nach Galen ...

4

Diese Feststellungen über die Grenzen von Platters Denken und Handeln dürften es verständlich machen, daß ihm die allgemeine Medizinhistorik bisher nur einen recht bescheidenen Platz in ihrer Ehrenhalle eingeräumt hat. Es fehlte Felix Platter die Kühnheit des großen Forschers, der aus dem, was er entdeckt hat, unbeirrbar seine Schlüsse zieht und seine neue, bessere Erkenntnis gegen alle Widerstände verficht. Auch als Archiater Basiliensis war er, wie wir gesehen haben, kein Kämpfer, selbst dort nicht, wo er es hätte sein sollen, wie angesichts der Pestgefahr. Kein großer Forscher, kein verbissener Kämpfer, sondern ein innig mit der antiken Tradition verbundener Renaissance-Mediziner - so charakterisiert, erscheint Platter wirklich nicht als eine jener heroischen Figuren, auf deren Häupter die Nachwelt so gern ihre Lorbeerkränze setzt.

Aber gerade in der Verwobenheit seines Denkens mit der Tradition scheint mir das Geheimnis von Platters Wirkung auf die Ärzte seiner Zeit und der nachfolgenden Generationen zu liegen. Diese Wirkung zeigte sich im Zustrom von Studenten und vor allem von Doktoranden nach Basel, solange Platter hier Professor war ${ }^{28}$, und sie zeigte sich nicht weniger in der Nachfrage nach seinen Schriften, vor allem nach der Praxis, die insgesamt fünf Auflagen erlebte, die letzte noch 1736. (Dazu kam 1662 noch eine englische Übersetzung ${ }^{29}$.) Daß Platter um die Mitte des 17.Jahrhunderts zu den maßgebenden medizinischen Autoritäten gerechnet wurde, läßt auch eine Leidener Ausgabe von Fernels Medicina universa aus dem Jahre $1645^{30}$ erkennen: Hier sind in die Pathologia Fernels zahlreiche Zitate aus

28 Siehe A.Burckhardt, op. cit. sub no. 4, Tabelle I, S. 428 f.

29 «Praxis» und «Observationes» Platters sind 1662 bzw. 1664 in englischer Übersetzung erschienen, s. P. Cranefield, Little known english versions of the Praxis and $\mathrm{Ob}-$ servationes of Felix Platter, J. Hist.Med. 17 (1962) 309-311.

30 J.Fernel, Universa medicina. Nova hac editione, quae obscura erant, illustrata, quae deficiebant, suppleta sunt (2 Bände), Leiden 1645 (Hackius). 
der Praxis Platters eingefügt. (Der Herausgeber Franciscus Hackius zitiert außerdem Dubors-Sylvius sowie die Niederländer Forestus und Heurnius.)

Gerade weil Platter gewisse Vorurteile seiner Zeitgenossen teilte, nahm man ihn ernst. Weil er beispielsweise die vermeintliche Besessenheit gewisser Irrer anerkannte, konnte er auch Gehör finden mit seiner Auffassung vom natürlichen Ursprung der meisten anderen Geisteskrankheiten. Weil er das umfassende galenische System der Pathologie und Therapie im groBen ganzen akzeptierte - und ein besseres gab es damals nicht -, konnte er es mit seinem kritischen Geist von manchen Auswüchsen spekulativer mittelalterlicher Medizin reinigen und vor allem den Vorrang der Beobachtung vor der Überlieferung darin zur Geltung bringen - sachte, aber nachhaltig. Vollkommen konsequent hat er, ein glühender Bewunderer VesALs, das anatomische Denken auf die praktische Heilkunde angewendet und damit der pathologischen Anatomie tatkräftig den Weg gebahnt.

Wenn wir Felix Platter nicht einfach leichthin von der Höhe unseres jetzigen Wissens aus beurteilen (auch diese Höhe ist ja nur relativ!), wenn wir vielmehr danach fragen, was er für seine Zeit gewesen ist, so erscheint er uns als derjenige Arzt und Gelehrte, der - die Renaissance-Periode der Medizin abschließend - ganz besonders geschickt und umsichtig das fruchtbare Neue, eben die Beobachtung in Anatomie und Klinik, mit dem noch unentbehrlichen Alten zu verbinden wußte. Er hat die unvermeidlicherweise noch galenische Medizin mit vesalischem Geist imprägniert. Er hat damit das gerade damals Notwendige getan und so den Ärzten, die mit ihm lebten und nach ihm kamen, weitergeholfen*.

* Während des Druckes sind zwei wichtige Aufsätze über Platter erschienen:

H. Buess, Der Einfluß Vesals auf die praktische Anatomie am Beispiel Felix Platters, Med. Monatsschr. 18 (1964) 502-507, und

Ingrid Schiewex, Zur Autobiographie des Basler Stadtarztes Felix Platter, Forsch. Fortschr. 38 (1964) 368-372. Frau Schiewek weist auf die innere Verwandtschaft der Autobiographie mit den «Observationes» hin, in denen Platters «spezifische schriftstellerische Begabung» ebenfalls zum Ausdruck kommt. 